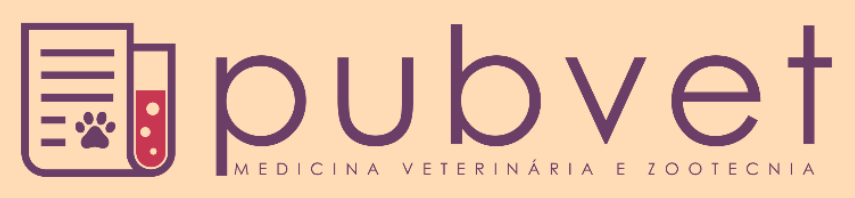

https://doi.org/10.31533/pubvet.v14n12a701.1-9

\title{
Estudo do efeito do gado na transmissão da malária na localidade de Massavasse distrito de Chókwè, província de Gaza, Moçambique
}

\author{
Venâncio Merique Nhavoto ${ }^{10}$ \\ ${ }^{I}$ Médico Veterinário, MSc. Maputo, Moçambique. Autor para correspondência, E-mail: vnhavoto@gmail.com
}

\begin{abstract}
Resumo. A zooprofilaxia é uma estratégia usada no controle da doença mediante o uso de animais, tendo-se mostrado eficaz em alguns países. Este trabalho objetivou avaliar o efeito do gado na transmissão da malária na localidade de Massavasse, distrito do Chóckwè. Mosquitos foram colhidos em casas com currais e sem currais durante a noite por armadilhas de luz e de tendas. A classificação das espécies foi feita pela chaves morfológicas, a determinação dos índices de infeção (IF) foi feita por ELISA e as diferenças entre casas com currais e sem currais testadas pelo teste $\chi^{2}$. Os resultados indicam não haver diferenças nos IF entre mosquitos do complex An. funestus encontrados juntos das casas com currais $(0,92 \% \pm 0,64 \% \mathrm{n}=217)$ e os de casas sem currais $(0,47 \% \pm$ $0,47 \% \mathrm{n}=211),(\mathrm{P}=0,51$; Teste exacto de Fisher $)$ capturados por armadilhas de tendas. $\mathrm{O}$ mesmo foi observado em relação os espécimes capturados por armadilhas de luz entre as casas com currais $(2,3 \% \pm 1,3 \% \mathrm{n}=130)$ e as de casas sem currais $(1,67 \% \pm 1,17 \% \mathrm{n}=$ 120) $(\mathrm{P}=0,54$; Teste exacto de Fisher). Nenhum dos 616 mosquitos do complex An. gambiae (An. arabiensis) foi encontrado positivo em casas com e sem currais. Entretanto, dos 678 mosquitos analisados da espécie An. funestus, 8 foram encontrados infectados. Estes resultados indicam que o An. funestus foi o principal vector da malária durante o período da realização deste estudo. $O$ fato de não se ter encontrado mosquitos de $A n$. arabiensis infectados sugere que estes possam se alimentar no gado. Contudo, estudos mais detalhados são necessários para avaliar o potencial do uso do gado para a zooprofilaxia da malária nas condições concretas de Moçambique.
\end{abstract}

Palavras chave: Malária, zooprofilaxia, gado, Anopheles arabiensis

\section{Study of the effect of cattle on malaria transmission in the locality of Massavasse - district of Chókwè, province of Gaza, Mozambique}

\begin{abstract}
Zooprophylaxis is a strategy used to control the disease upon the use of animals and has been shown to be effective in some countries. This work aimed to evaluate the effect of cattle on malaria transmission in the locality of Massavasse, district of Chóckwè. Mosquitoes were collected in houses with corrals and without corrals at night using light traps and tent traps. Species were classified using morphological keys, infection rates (IR) were determined using an ELISA and differences between houses with corrals and without corrals were statistically analyzed by $\chi^{2}$ test. The results show that significant statistical differences do not exist in the infection rates between the mosquitoes of the An. funestus complex from the houses with corrals $(0.92 \% \pm 0.64 \% \mathrm{n}=217)$, the houses without corrals $(0.47 \% \pm 0.47 \% \mathrm{n}=211)$, and $(\mathrm{P}=0.51$; Fisher's exact test $)$ captured through tent traps. The same was observed pertaining to the specimens captured through light traps between houses with corrals $(2.3 \% \pm 1.3 \% \mathrm{n}=130)$ and houses without corrals $(1.67 \% \pm 1.17 \% \mathrm{n}$ $=120)(\mathrm{P}=0.54 ;$ Fisher's exact test $)$. None of the 616 mosquitoes of the An. gambiae complex (An. arabiensis) were found positive in houses with or without a corral. However,
\end{abstract}


of the 678 analyzed mosquitoes of the species An. funestus, 8 were found infected. These results indicate that An. funestus was the main malaria vector during the period of this study. The fact that any infected An. arabiensis mosquitoes were found suggest that they can feed on cattle. However, more detailed studies are needed to assess the potential use of cattle for malaria zooprophylaxis under the specific conditions of Mozambique.

Keywords: Malaria, zooprophylaxis, cattle, Anopheles arabiensis

\section{Introdução}

A malária humana é transmitida por mosquitos do género Anopheles (An.) e a forma mais grave é a causada pelo parasita Plasmodium falciparum (Murray et al., 1978). A sua distribuição é influenciada pela temperatura atmosférica, precipitação e humidade. Estes fatores actuam directamente no vector providenciando locais de criação e prolongando a vida do vector adulto (Symes et al., 1962). A humidade, tem um efeito marcado na taxa de sobrevivência dos mosquitos (Clyde, 1987). Algumas espécies de anofelinos constituem complexos, sendo que o complexo An. gambiae compreende seis espécies biológicas dentre as quais An. gambiae e An. arabiensis são tidas como sendo os vectores mais eficientes da malária em África (Coluzzi et al., 1979; White, 1974). O complexo An. funestus compreende pelo menos oito espécies (M T Gillies \& Coetzee, 1987). Na região de Chóckwè, as únicas espécies encontradas do complexo An. gambiae foram o An. arabiensis (87,0\%) e An. merus (13,0\%) (Petrarca et al., 1984), corroborado posteriormente por Cuamba et al. (1996). O grande crescimento populacional no continente Africano tem conduzido a crescente carência de alimentos e tendo em vista a superar esta necessidade, muitos países têm procurado meios de melhorar a produção implementando projectos de irrigação em larga escala. Contudo, a irrigação é tida como um fator responsável pelo agravamento de malária (Ijumba \& Lindsay, 2001). Estudos efetuados em zonas irrigadas de cultivo de arroz e em zonas não irrigadas no Mali mostraram diferenças nítidas na transmissão da malária. Na zona irrigada verificou-se uma alta densidade de mosquitos e a transmissão da malária foi constante durante as estações do ano mantendo-se a um nível baixo, enquanto que nas zonas não irrigadas a densidade de mosquitos foi baixa. A transmissão da malária foi baixa durante a época seca, mas alta na estação chuvosa. A presença do gado na zona irrigada pode ter desempenhado um papel importante nos níveis baixos de transmissão da doença (Dolo et al., 2004). A zooprofilaxia refere-se ao controlo do vetores desviando-os para animais domésticos nos quais o patógeno não se pode multiplicar. Contudo, em África existe pouca informação sobre o assunto (Bøgh et al., 2002; Saul, 2003) e nas condições de Moçambique não existe informação do impacto de zooprofilaxia sobre a transmissão da malária. Se a zooprofilaxia pode funcionar em relação as condições de Moçambique, a criação do gado bovino nas comunidades rurais pode ser aproveitada como forma de reduzir o risco de infecção por malária na localidade de Massavasse.

\section{Material e métodos}

\section{Área de estudo}

Este estudo foi realizado na localidade de Massavasse, distrito de Chókwè, província de Gaza, Moçambique. O distrito de Chókwè é potencialmente rico em recursos agrícolas, possui solos férteis para o cultivo de variedades de culturas. Possui ainda, infraestruturas de irrigação e é um dos principais produtores de bovinos em Moçambique (UNDP \& UNHCR, 1997). O clima da região é semi-árido a seco. Massavasse situa-se no regadio do vale do Limpopo (24 $37^{\prime} \mathrm{S} 33^{\circ} 06^{\prime} \mathrm{E}$ ) e tem cerca de 5.000 habitantes distribuídos em 1.300 casas encontradas em 5 bairros, sendo um terço das casas feitas de caniço, um terço são construídas com estacas, argila e um terço de alvenaria. O número médio dos habitantes varia entre os tipos de casas, sendo seis em casas de alvenaria e três nos outros tipos. A agropecuária é a atividade econômica mais importante entre os residentes de Massavasse, destacando-se o cultivo de arroz e hortícolas e os criadores têm a tendência de fixar os currais junto das habitações humanas. A precipitação média anual é de $622 \mathrm{~mm}$, atingindo o valor máximo de $140 \mathrm{~mm}$ em fevereiro e o mínimo de $10 \mathrm{~mm}$ em julho, a época chuvosa vai de outubro a março e a seca de abril à setembro (FAO, 1984). A média mensal da temperatura varia de $18,5^{\circ} \mathrm{C}$ no mês de junho e julho a $27^{\circ} \mathrm{C}$ nos meses quentes, (dezembro a fevereiro), a média anual é de $23,6{ }^{\circ} \mathrm{C}$ (Faculdade de Agronomia e Engenharia Florestal, 2001). 


\section{Colheita dos mosquitos}

A colheita de mosquitos, vetores de malária foi realizada durante a estação chuvosa, entre os meses de fevereiro a abril dentro e fora das habitações. Dentro das habitações os mosquitos foram colhidos durante a noite com uso de armadilhas de luz do tipo CDC Atlanta e fora destas com uso de "tent-trap" segundo o método de Charlwood (não publicado). As colheitas foram feitas em casas construídas a base de material local, nomeadamente estacas, caniço, palha e "maticadas" com lodo e fezes de bovinos. Durante a noite, procedia-se a captura de mosquitos e efetuava-se a montagem de redes mosquiteiras nas casas que não possuíam redes com o objetivo de se obter as mesmas condições experimentais. Para avaliar a quantidade de vectores nas habitações e comparação dos níveis de infeção dos vectores de malária sete pares de armadilhas de tenda e sete pares de armadilhas de luz foram colocadas nos bairros de Massavasse, em habitações selecionadas que possuíam currais nos quintais e habitações sem currais. $\mathrm{Na}$ Figura 1 pode observar-se vetores de malária na cabeça de um bovino. As armadilhas de tenda eram ativadas e retiradas pelo coletor a mesma hora que as armadilhas de luz. Após a identificação morfológica e contagem dos mosquitos estes eram conservados em tubos Eppendorf contendo gel de sílica e algodão, na parte inferior do tubo.

\section{Armadilhas de luz}

As armadilhas de luz foram colocadas no interior das habitações no final da tarde simultaneamente com a montagem das armadilhas de tenda e os moradores faziam a ligação da bateria as $20 \mathrm{~h}$, desligando as $5 \mathrm{~h}$ da manhã e amarrando o saco da armadilha (Figura 2) contendo mosquitos. Estas armadilhas consistem numa tampa de protecção e fixação, uma bateria para a emissão de luz e funcionamento de uma ventoínha que permitia a sução dos mosquitos para um cilindro e posteriormente um saco de recolha, onde os mosquitos se acumulavam.

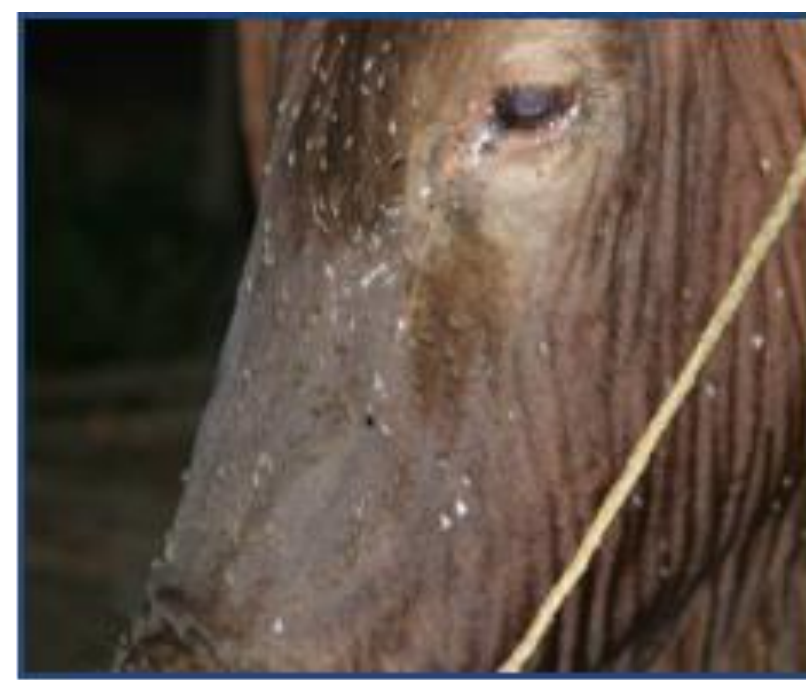

Figura 1. Parte da cabeça de um bovino infestada por mosquitos, alguns dos quais vetores de malária.

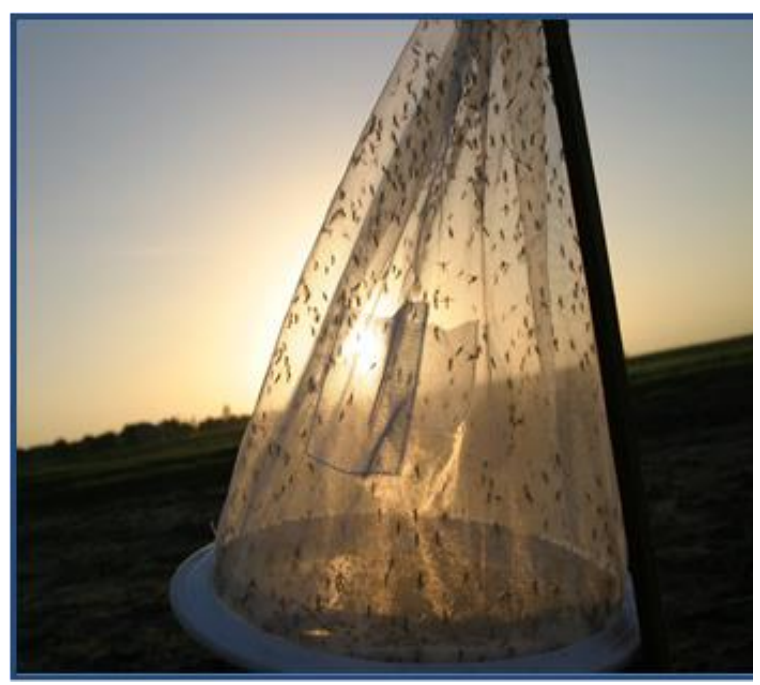

Figura 2. Mosquitos no saco da armadilha de luz.

\section{Armadilhas de tenda}

As armadilhas de tenda usadas para a captura de mosquitos fora das habitações, consitem numa tenda de campo com uma abertura que permitia a libertação do odor do hospedeiro humano para o exterior. Próximo a entrada da tenda era fixada a ventoínha de sução que funcionava através da bateria, permitindo a ventilação necessária para que os mosquitos fossem sugados para o saco. As tendas eram apenas ocupadas por um hospedeiro humano.

\section{Identificação de mosquitos}

Depois da colheita e ainda no campo, a separação de mosquitos em membros dos complexos $A n$. gambiae e An. funestus foi feita através de métodos morfológicos descritos em Gillies \& De Meillon (1970) e Gillies \& Coetzee (1987). 


\section{Determinação dos índices de infecção dos vectores}

Os índices de infeção dos mosquitos foram determinados através da técnica ELISA de acordo com Burkot et al. (1984). Esta técnica é um ELISA do tipo sanduíche que permite detectar circumsporozoítos de $P$. falciparum. As amostras de mosquitos foram preparadas separando o abdómen e triturando a cabeça e tórax de mosquitos individuais em tampão de bloqueio (consistindo de PBS, caseína fervida com adição de Nonidet P-40 e vermelho de fenol) sendo armazenadas de seguida em frio $\left(-20^{\circ} \mathrm{C}\right)$ para o processamento, de acordo com os procedimentos do teste.

\section{Análise de dados}

O número total de mosquitos identificados colhidos nas armadilhas de tenda e de luz, foi registado diariamente nos formulários de recolhas entomológicas. Ainda no campo, os dados foram introduzidos na folha de cálculo do Microsoft Excel $^{\circledR}$ contendo informações como número da casa, data, local e método de colheita. Os dados entomológicos foram manuseados usando o Microsoft Excel ${ }^{\circledR}$ para obter o número total de mosquitos capturados no interior das habitações (armadilha de luz) e no exterior (armadilhas de tenda) próximo dos currais. O teste qui-quadrado $\left(\chi^{2}\right)$ foi executado no programa EpiInfo 6.0 versão 2000 para medir a significância das diferenças entre os índices de infeção dos mosquitos encontrados em casas com currais e as que não tinham. O nível de significância aplicado foi de $\mathrm{P}<0,05$.

\section{Resultados}

\section{Mapeamento dos locais de captura de vectores}

O mapeamento da localidade de Massavasse e dos locais de colheita dos vetores através dos dois métodos usados anteriormente em casas com currais e em casas sem currais são mostrados na figura 3. Todas as casas e os locais de montagem das armadilhas (representado por triângulos) foram situadas utilizando o GPS (Global Positioning System) e os dados introduzidos no Microsoft Excel ${ }^{\circledR}$ para posterior criação do mapa.

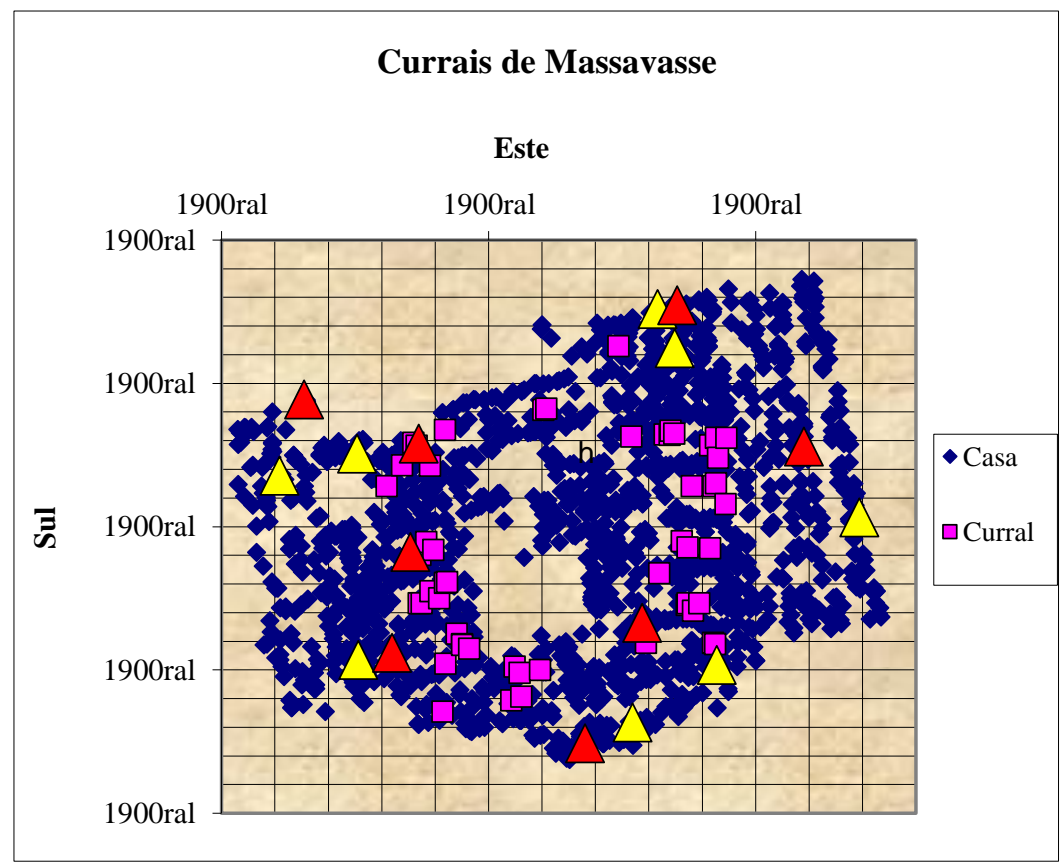

Figura 3. Mapa da localidade de Massavasse feito através de GPS, mostrando os locais de captura de vetores em casas com currais e sem currais.

\section{Abundância de vetores de malária nas habitações}

A tabela 1 mostra a comparação de números totais de vetores de malária por método de colheita e em função da presença do gado. Os resultados indicam não haver diferenças na quantidade de vetores do complexo An. gambiae de casas com currais e sem currais $\left(\chi^{2}=1,61 ; \mathrm{df}=1 ; \mathrm{P}>0,05\right)$. Quanto ao 
complexo An. funestus não foram também observadas diferenças significativas na quantidade de vetores capturados em casas com currais e sem currais $\left(\chi^{2}=1,87\right.$;f $\left.=1 ; \mathrm{P}>0,05\right)$.

Tabela 1. Comparação da quantidade de vetores em função do método de colheita e da presença de currais

\begin{tabular}{lccccc}
\hline \multirow{2}{*}{ Armadilha } & \multicolumn{2}{c}{ An. funestus } & & \multicolumn{2}{c}{ An. gambiae } \\
\cline { 2 - 3 } \cline { 5 - 6 } & Com curral & Sem curral & & Com curral & Sem curral \\
\hline Tenda & 204 & 286 & 280 & 346 \\
Luz & 147 & 169 & 54 & 85 \\
Total & 351 & 455 & 334 & 431 \\
\hline
\end{tabular}

\section{Índices de infeção dos vetores}

As reacções positivas do teste ELISA resultaram numa cor verde na placa. Todos os espécimes encontrados positivos perteciam ao complexo An. funestus. Não se observou nenhum vetor infectado do complexo An. gambiae. Os índices esporozoíticos dos vetores pelo método de colheita e em função da presença do gado são mostrados na tabela 2. Dos anofelinos do complexo An. funestus capturados através da técnica de armadilhas de luz, 0,92\% (2/217) foram positivos em casas com currais e 0,47\% $(1 / 211)$ em casas sem currais, estes resultados não mostram diferenças significativas $(\mathrm{P}=0,51$; Teste exacto de Fisher). Pela técnica de armadilhas de tenda 2,3\% (3/130) foram positivos em casas com currais e 1,67\% (2/120) provenientes de casas sem currais, não tendo-se também observado diferenças significativas $(\mathrm{P}=0,54 ;$ Teste exacto de Fisher $)$.

Tabela 2. Índices esporozoíticos dos vetores em função do método de colheita e da presença de currais em Massavasse. Os valores entre parênteses indicam o intervalo de confiança.

\begin{tabular}{|c|c|c|c|c|c|c|}
\hline \multirow{2}{*}{$\begin{array}{l}\text { Método de } \\
\text { colheita }\end{array}$} & \multicolumn{3}{|c|}{ An. funestus } & \multicolumn{3}{|c|}{ An. gambiae } \\
\hline & $\mathrm{N}^{\circ}$ de examinados & Infectados & $\%$ & $\mathrm{~N}^{\circ}$ de examinados & Infectados & $\%$ \\
\hline Luz com curral & 217 & 2 & $0,92(0,92 \pm 0,64)$ & 76 & 0 & 0 \\
\hline Luz sem curral & 211 & 1 & $0,47(0,47 \pm 0,47)$ & 176 & 0 & 0 \\
\hline Tenda com curral & 130 & 3 & $2,3(2,3 \pm 1,3)$ & 198 & 0 & 0 \\
\hline Tenda sem curral & 120 & 2 & $1,67(1,67 \pm 1,17)$ & 166 & 0 & 0 \\
\hline Total & 678 & 8 & $1,18(1,18 \pm 0,41)$ & 616 & 0 & 0 \\
\hline
\end{tabular}

Os índices de infeção total de mosquitos de casas com currais e sem currais foram somados e mostrados na tabela 3 . Os resultados indicam não haver diferenças estatisticamente significativas entre vetores do complexo An. funestus de casas com currais e sem currais, ( $\mathrm{P}=0,39$; Teste exacto de Fisher).

Tabela 3. Índices de infeção total dos vetores capturados em casas com currais e sem currais na localidade de Massavasse

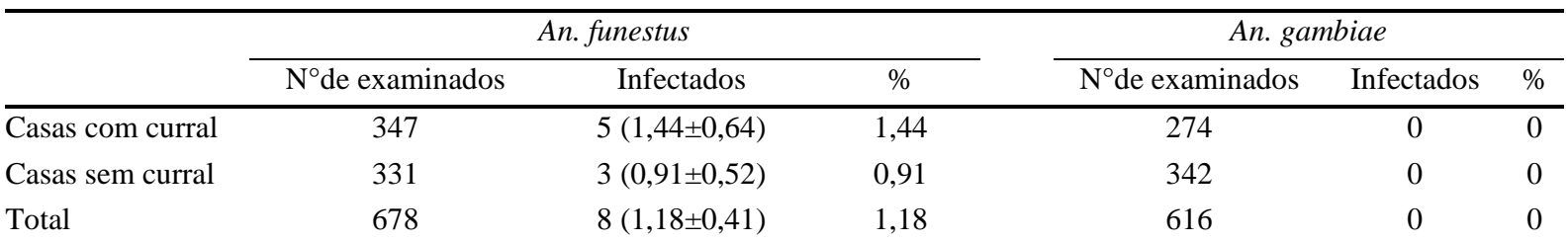

\section{Discussão}

O objetivo da luta antivetorial com métodos tradicionais (pulverizações, rede mosquiteiras), na malária é geralmente reduzir o número de mortes através da redução da probabilidade de sobrevivência diária. A zooprofilaxia é um método de luta antivetorial que visa a redução da transmissão da malária pela redução do contato homem-vetor. Alguns anofelinos têm marcada preferência pelo sangue humano, escolhendo-o sempre que possível, outros têm grande preferência por animais e raramente alimentamse em humanos, não sendo deste modo usual que estes transmitam malária (Gilles \& Warrell, 1996). Esta preferência pelo sangue animal (zoofilia) tem sido aproveitada como um valioso instrumento no controlo da malária (zooprofilaxia) como já foi observado em algumas regiões, como por exemplo na Ilha de Java (Kirnowordoyo, 1986). Na Itália, por exemplo, o An. messeae era zoofílico e não associado 
com a malária, mas no Este da Europa e na União Soviética após a segunda guerra Mundial a espécie foi considerada um importante vetor da doença. $\mathrm{O}$ aumento da antropofilia desta espécie foi atribuída a drástica redução de animais domésticos como resultado da guerra (Kettle, 1984). Outro exemplo, é do complexo An. minimus cujas populações mostraram diferenças intraespecíficas no comportamento. No Norte de Vietname, a espécie A deste complexo tendeu a ser mais zoofílica, enquanto que no Sul e Centro do país, Cambodja e Lagos, o An. minimus A mostrou acentuado comportamento antropofílico quando o gado bovino era escasso (Van Bortel et al., 2004). De acordo com Donnelly et al. (2015) a zooprofilaxia pode ser parte de uma estratégia eficaz para reduzir a transmissão da malária sob condições ecológicas e geográficas específicas. Por exemplo, na Indonésia, Hasyim et al. (2018) verificaram que manter o gado em casa contribui para o risco de malária, em vez de profilaxia. O comportamento dos mosquitos Anopheles arabiensis varia entre localidades e circunstâncias, assim sugere-se uma avaliação específica do local para a eficácia da zooprofilaxia (Asale et al., 2017).

A abundância dos anofelinos é influenciada por dois fatores principais, a temperatura e a disponibilidade de locais de criação (Kettle, 1984). Contudo, os resultados deste trabalho indicam que não existem diferenças estatisticamente significativas na quantidade de vetores de malária entre casas com currais e sem currais. Isto deve estar relacionado com os métodos de colheita aplicados. De fato, as duas técnicas de colheita utilizadas capturam aqueles mosquitos na altura em que estes procuram a refeição sanguínea. Contudo, quando se trata de zooprofilaxia, o mais importante é saber se os mosquitos se alimentam de fato com sangue humano ou com o sangue de animais ou através da comparação dos índices de infeção em casas com animais ou sem estes. Na localidade de Massavasse não foram encontrados mosquitos do complexo An. gambiae infectados com $P$. falciparum, a espécie predominante deste complexo é o An. arabiensis (Cuamba et al., 1996; Cuamba \& Mendis, 2009). Como referiu-se anteriormente, os índices sanguíneos funcionam como indicador válido na utilidade da zooprofilaxia, pois os melhores vetores de malária são em regra aqueles que se alimentam de sangue humano. Espécies que se alimentam em diferentes hospedeiros não são bons vetores como espécies altamente antropofágicas (Githeko et al., 1994). No estudo de preferências alimentares dos mosquitos, espera-se baixos índices de sangue humano, como observado em estudos realizados em Mwea no centro de Kenya. Os resultados do estudo do Kenya, mostraram que onde existia gado os índices de sangue humano na espécie An. arabiensis eram baixos $(0,1)$ e em Antananarivo, área de Madagascar nenhuma fêmea foi encontrada alimentando-se em humanos, em local com gado (Ijumba et al., 1990). Em Gambela, Etiópia onde nenhum bovino se encontrava na proximidade das casas, foram encontradas fêmeas de An. arabiensis alimentando-se somente em humanos (Krafsur, 1971). Resultados obtidos na vila de Mwea, Kenya, demonstraram que a presença do gado e o cultivo de arroz estão relacionados com a malária. Nesta vila, An. arabiensis teve tendência de se alimentar mais no gado e as altas frequências de sangue bovino no mosquito resultaram em baixa prevalência de malária na comunidade (Mutero et al., 2004). No mesmo estudo, observou-se que a diminuição do gado nos esquemas de irrigação pode por outro lado resultar em consequências desastrosas com aumento de casos de malária devido ao fato do An. arabiensis retornar ao hospedeiro humano a busca de sangue. Também no Kenya, nas plantações de cana de açúcar da localidade de Mwani, onde o gado estava ausente, os trabalhadores eram a única fonte de sangue e consequentemente a transmissão de malária foi maior (Githeko et al., 1994). De acordo com Waka et al. (2005) são observadas altas taxas de zoofagia no An. arabiensis quando existem disponíveis mamíferos. Muitos animais, podem reduzir o tempo gasto pelos mosquitos a procura de refeição de sangue com correspondente reduzida mortalidade na procura de sangue (Saul, 2003), e ao se alimentarem de sangue animal reduz-se a taxa reprodutiva do parasita de malária, porque os vetores são mais atraídos para o hospedeiro de morte (Atkinson et al., 2003; Kawaguchi et al., 2004). O fato de nenhum vetor do complexo An. gambiae (An. arabiensis) ter sido encontrado infetado através do teste ELISA, sugere que este vetor alimenta-se de sangue no gado bovino com consequente perda de capacidade vetorial. An. arabiensis é geralmente considerado como sendo prontamente desviado para alimentar-se no gado (Githeko et al., 1994). Em contraste, apesar da presença de animais nos currais próximo as habitações de Massavasse anofelinos do complexo An. funestus apresentaram índices esporozoíticos aproximados nas casas com currais e sem currais, o que indica que espécies deste complexo dificilmente são atraídas pelo gado, não tendo-se registado grande perturbação da atração de mosquitos ao homem devido a forte antropofilia de vetores deste complexo. Estes resultados indicam que a zooprofilaxia pode ter efeito para as condições de Massavasse, no concernante aos mosquitos do complexo An. gambiae. Isto pode explicar o fato de nenhum mosquito ter sido encontrado 
infetado. O efeito de massa, isto é, a densidade do gado na localidade é elevada e os currais distribuem-se pelos bairros de tal modo que existe a possibilidade dos vetores que se encontravam na área tenham sido atraídos ao contato com o gado. Contudo, estudos longitudinais, envolvendo um número elevado de colheitas, deverão confirmar estes resultados. Estes estudos deverão incluir além dos índices esporozoíticos, os índices de sangue humano. Isto poderá melhor explicar os índices de infeção nulos nos vetores do complexo An. gambiae (arabiensis). Assumindo, os resultados, a criação do gado bovino nas comunidades rurais poderá ser também aproveitada como forma de reduzir o risco de infeção por malária.

\section{Conclusão}

A presença do gado bovino sugere zooprofilaxia contra anofelinos do complexo An. gambiae (An. arabiensis) na localidade de Massavasse, assim o risco de contrair malária pode ser reduzido, já que apenas vetores do complexo An. funestus foram encontrados infetados. Contudo, outros métodos suplementares são recomendados para reduzir o risco por An. funestus, tais como a colocação de redes mosquiteiras, eliminação de abrigos naturais tais como jarros de louça de barro e barris de madeira, através de limpeza no interior das habitações e acima de tudo, as pulverizações intradomiciliárias impedindo que muitos vetores sobrevivam o suficiente para transmitirem os esporozoítos. Estudos posteriores poderão confirmar o efeito de zooprofilaxia em An. arabiensis. Apesar de não se ter detetado anofelinos do complexo An. gambiae (An. arabiensis) infetados, sugere-se estudos em outros locais de criação do gado próximo as habitações.

\section{Agradecimentos}

Ao Dr. Nelson Jacinto Bambo Cuamba, Dr. Jacques Derek Charlwood pelo delineamento e acompanhamento do trabalho de campo, Dr. Luís Carlos Bernardo Gil Neves, Dr. Alberto Pondja, Dr. Patrícia Ferrara e ao DBL-students (Danish Bilharziasis Laboratory) pelo financiamento, aos coletores e técnicos de laboratório.

\section{Referências bibliográficas}

Asale, A., Duchateau, L., Devleesschauwer, B., Huisman, G., \& Yewhalaw, D. (2017). Zooprophylaxis as a control strategy for malaria caused by the vector Anopheles arabiensis (Diptera: Culicidae): a systematic review. Infectious Diseases of Poverty, 6(1), 160. DOI: https://doi.org/10.1186/s40249017-0366-3

Atkinson, D., Ciotti, B. J., \& Montagnes, D. J. S. (2003). Protists decrease in size linearly with temperature: ca. $2.5 \%$ C- 1. Proceedings of the Royal Society of London. Series B: Biological Sciences, 270(1533), 2605-2611. DOI: https://doi.org/10.1098/rspb.2003.2538

Bøgh, C., Clarke, S. E., Walraven, G. E. L., \& Lindsay, S. W. (2002). Zooprophylaxis, artefact or reality? A paired-cohort study of the effect of passive zooprophylaxis on malaria in The Gambia. Transactions of the Royal Society of Tropical Medicine and Hygiene, 96(6), 593-596. DOI: https://doi.org/10.1016/S0035-9203(02)90320-2

Burkot, T. R., Williams, J. L., \& Schneider, I. (1984). Identification of Plasmodium falciparum-infected mosquitoes by a double antibody enzyme-linked immunosorbent assay. The American Journal of Tropical Medicine and Hygiene, 33(5), 783-788. DOI: https://doi.org/10.4269/ajtmh.1984.33.783

Clyde, D. F. (1987). Recent trends in the epidemiology and control of malaria. Epidemiologic Reviews, 9(1), 219-243. DOI: https://doi.org/10.1093/oxfordjournals.epirev.a036303

Coluzzi, M., Sabatini, A., Petrarca, V., \& Di Deco, M. A. (1979). Chromosomal differentiation and adaptation to human environments in the Anopheles gambiae complex. Transactions of the Royal Society of Tropical Medicine and Hygiene, 73(5), 483-497. DOI: https://doi.org/10.1016/00359203(79)90036-1

Cuamba, N, Theron, D., \& Dgedge, M. (1996). Contribuição ao conhecimento da distribuição do complexo Anopheles gambiae em Moçambique a Sul da latitude $19^{\circ}$. Revista Médica de Moçambique, 6, 5-8.

Cuamba, Nelson, \& Mendis, C. (2009). The role of Anopheles merus in malaria transmission in an area of southern Mozambique. Journal of Vector Borne Diseases, 46(2), 157-159. 
Dolo, G., Briët, O. J. T., Dao, A., Traoré, S. F., Bouaré, M., Sogoba, N., Niaré, O., Bagayogo, M., Sangaré, D., \& Teuscher, T. (2004). Malaria transmission in relation to rice cultivation in the irrigated Sahel of Mali. Acta Tropica, 89(2), 147-159. DOI: https://doi.org/10.1016/j.actatropica.2003.10.014

Donnelly, B., Berrang-Ford, L., Ross, N. A., \& Michel, P. (2015). A systematic, realist review of zooprophylaxis for malaria control. Malaria Journal, 14(1), 313. DOI: https://doi.org/10.1186/s12936-015-0822-0

Faculdade de Agronomia e Engenharia Florestal (2001). Programa Competir-Diagnóstico da fileira agrícola. Universidade Eduardo Mondlane. Maputo, Moçambique.

FAO (1984). Agroclimatological data of Africa, vol. 2. Plant and protection series.

Gilles, H. M., \& Warrell, D. A. (1996). Bruce-Chwatt's essential malariology (Issue Ed. 3). Edward Arnold (Publisher) Ltd.

Gillies, M T, \& Coetzee, M. (1987). A supplement to the Anophelinae of Africa South of the Sahara. The South African Institute for Medical Research, 55, 1-143.

Gillies, Michael Thomas, \& De Meillon, B. (1970). The Anophelinae of Africa south of the Sahara (Ethiopian zoogeographical region). The Anophelinae of Africa South of the Sahara, 19(4), 736-736. DOI: https://doi.org/10.4269/ajtmh.1970.19.4.tm0190040736a

Githeko, A. K., Service, M. W., Mbogo, C. M., Atieli, F. K., \& Juma, F. O. (1994). Origin of blood meals in indoor and outdoor resting malaria vectors in western Kenya. Acta Tropica, 58(3-4), 307316. DOI: https://doi.org/10.1016/0001-706X(94)90024-8

Hasyim, H., Dhimal, M., Bauer, J., Montag, D., Groneberg, D. A., Kuch, U., \& Müller, R. (2018). Does livestock protect from malaria or facilitate malaria prevalence? A cross-sectional study in endemic rural areas of Indonesia. Malaria Journal, 17(1), 302. DOI: https://doi.org/10.1186/s12936-018-2447-6

Ijumba, J. N., \& Lindsay, S. W. (2001). Impact of irrigation on malaria in Africa: paddies paradox. Medical and Veterinary Entomology, 15(1), 1-11. DOI: https://doi.org/10.1046/j.13652915.2001.00279.x

Ijumba, J. N., Mwangi, R. W., \& Beier, J. C. (1990). Malaria transmission potential of Anopheles mosquitoes in the Mwea-Tebere irrigation scheme, Kenya. Medical and Veterinary Entomology, 4(4), 425-432. DOI: https://doi.org/10.1111/j.1365-2915.1990.tb00461.x

Kawaguchi, I., Sasaki, A., \& Mogi, M. (2004). Combining zooprophylaxis and insecticide spraying: a malaria-control strategy limiting the development of insecticide resistance in vector mosquitoes. Proceedings of the Royal Society of London. Series B: Biological Sciences, 271(1536), 301-309. DOI: https://doi.org/10.1098/rspb.2003.2575

Kettle, D. S. (1984). Medical and veterinary entomology. Croom Helm Ltd. DOI: https://doi.org/10.1653/024.102.0321

Kirnowordoyo, S. (1986). Zooprophylaxis as a useful tool for control of A. aconitus transmitted malaria in Central Java, Indonesia. Journal of Communicable Diseases, 18(2), 90-94.

Krafsur, E. S. (1971). Malaria transmission in Gambela, Illubabor province. Ethiopian Medical Journal, 9(2), 75-94.

Murray, J., Murray, A., Murray, M., \& Murray, C. (1978). The biological suppression of malaria: an ecological and nutritional interrelationship of a host and two parasites. The American Journal of Clinical Nutrition, 31(8), 1363-1366. DOI: https://doi.org/10.1093/ajen/31.8.1363

Mutero, C. M., Kabutha, C., Kimani, V., Kabuage, L., Gitau, G., Ssennyonga, J., Githure, J., Muthami, L., Kaida, A., \& Musyoka, L. (2004). A transdisciplinary perspective on the links between malaria and agroecosystems in Kenya. Acta Tropica, 89(2), 171-186. DOI: https://doi.org/10.1016/j.actatropica.2003.07.003

Petrarca, V., Carrara, G. C., Di Deco, M. A., \& Petrangeli, G. (1984). Osservazioni citogenetiche e biometriche sui membri del complesso Anopheles gambiae in Mozambico. Parassitologia, 26(3), 247-259.

Saul, A. (2003). Zooprophylaxis or zoopotentiation: the outcome of introducing animals on vector transmission is highly dependent on the mosquito mortality while searching. Malaria Journal, 2(1), 32. DOI: https://doi.org/10.1186/1475-2875-2-1 
Symes, C. B., Muirhead Thompson, R. C., \& Busvine, J. R. (1962). Insect control in public health. In Insect control in public health. Elsevier Publishing Co. DOI: https://doi.org/10.4269/ajtmh.1963.12.3.tm0120030444a

UNDP \& UNHCR (1997). District Development Profiles - Chókwè District. Maputo, Moçambique.

Van Bortel, W., Trung, H. D., Sochantha, T., Keokenchan, K., Roelants, P., Backeljau, T., \& Coosemans, M. (2004). Eco-ethological heterogeneity of the members of the Anopheles minimus complex (Diptera: Culicidae) in Southeast Asia and its consequences for vector control. Journal of Medical Entomology, 41(3), 366-374. DOI: https://doi.org/10.1603/0022-2585-41.3.366

Waka, M., Hopkins, R. J., Akinpelu, O., \& Curtis, C. (2005). Transmission of malaria in the Tesseney area of Eritrea: parasite prevalence in children, and vector density, host preferences, and sporozoite rate. Journal of Vector Ecology, 30(1), 27-32.

White, G. B. (1974). Anopheles gambiae complex and disease transmission in Africa. Transactions of the Royal Society of Tropical Medicine and Hygiene, 68(4), 278-298. DOI: https://doi.org/10.1016/0035-9203(74)90035-2.

Histórico do artigo:

Recebido: 10 de agosto, 2020.

Aprovado: 1 de setembro, 2020.

Disponível online: 9 de novembro, 2020.
Licenciamento: Este artigo é publicado na modalidade Acesso Aberto sob a licença Creative Commons Atribuição 4.0 (CC-BY 4.0), a qual permite uso irrestrito, distribuição, reprodução em qualquer meio, desde que o autor e a fonte sejam devidamente creditados. 